\title{
Phase-coexisting patterns, horizontal segregation, and controlled convection in vertically vibrated binary granular mixtures
}

\author{
Istafaul Haque Ansari, ${ }^{1}$ Nicolas Rivas, ${ }^{2,3}$ and Meheboob Alam ${ }^{1, *}$ \\ ${ }^{1}$ Engineering Mechanics Unit, Jawaharlal Nehru Centre for Advanced Scientific Research, Jakkur PO, Bangalore 560064, India \\ ${ }^{2}$ Multi-Scale Mechanics, MESA+, University of Twente, Enschede, Netherlands \\ ${ }^{3}$ Forschungszentrum Jülich GmbH, Helmholtz-Institut Erlangen-Nürnberg für Erneuerbare Energien (IEK-11), \\ Fürther Strasse 248, 90429 Nuremberg, Germany
}

(Received 2 November 2017; published 26 January 2018)

\begin{abstract}
We report patterns consisting of coexistence of synchronous and asynchronous states [for example, a granular gas co-existing with (i) bouncing bed, (ii) undulatory subharmonic waves, and (iii) Leidenfrost-like states] in experiments on vertically vibrated binary granular mixtures in a Hele-Shaw cell. Most experiments have been carried out with equimolar binary mixtures of glass and steel balls of same diameter by varying the total layer height $(F)$ for a range of shaking acceleration $(\Gamma)$. All patterns as well as the related phase diagram in the $(\Gamma, F)$ plane have been reproduced via molecular dynamics simulations of the same system. The segregation of heavier and lighter particles along the horizontal direction is shown to be the progenitor of such phase-coexisting patterns as confirmed in both experiment and simulation. At strong shaking we uncover a partial convection state in which a pair of convection rolls is found to coexist with a Leidenfrost-like state. The crucial role of the relative number density of two species on controlling the buoyancy-driven granular convection is demonstrated. The onset of horizontal segregation can be explained in terms of an anisotropic diffusion tensor.
\end{abstract}

DOI: 10.1103/PhysRevE.97.012911

\section{INTRODUCTION}

Real particles are always polydisperse, the simplest case being a binary mixture of particles of different density and/or size. A binary granular mixture driven by external vibrations is known to lead to spontaneous segregation or demixing [1] of two species, which can be a nuisance or a blessing for industries dealing with granular materials. The segregation in binary mixtures has been extensively studied under vertical and horizontal vibration [2] as well as in different shearing experiments [3], although a unified theory for Brazil-nut segregation [4] and its reverse [5-7] is still missing. Understanding segregation-induced patterns can help to control and manipulate particulate flows, for example, in chemical and pharmaceutical industries. Moving away from applications, patterns are important in dynamical systems theory and nonequilibrium statistical mechanics, since they provide an avenue to probe the validity of any self-consistent theory. Understanding segregation-driven patterns and finding ways to control them is a key challenge in granular physics research.

A vertically shaken box of particles displays a rich variety of patterns: Faraday-type sub-harmonic waves and heaping [8-10], oscillon [11], convection [12-15], Leidenfrost-like density inversion [16,17], and segregation [18,19], with each pattern being characterized by its distinct spatial and temporal symmetries. While patterns having different spatial symmetry are routinely found to coexist (e.g., the gas, liquid, and solid

*meheboob@jncasr.ac.in coexists in driven granular matter [20,21]), the coexistence of patterns with different temporal symmetry (e.g., a subharmonic wave coexisting with a harmonic or an asynchronous state) is rare. An example of the latter-type patterns is the wellknown "oscillon" [11] which was discovered in Faraday-type experiments on granular materials under vertical shaking - this represents a period- 2 state that coexists with a period- 1 bouncing bed. Such patterns having different spatial and temporal symmetries are heretofore dubbed "phase-coexisting" patterns. For monodisperse granular particles under harmonic shaking, we refer to recent works in Refs. [14,22,23] for a broad overview of all observed patterns as well as contributions of different groups; the present work is focused on uncovering patterns in binary granular mixtures in a Hele-Shaw-type container under vertical shaking.

In this paper, a variety of patterns, having different spatial and temporal symmetries (one example being a period-2 wave coexisting with a disordered and asynchronous granular gas), is uncovered in vertically shaken binary granular mixtures that challenges current theoretical understanding of granular flows. It is shown that the "buoyancy-induced" granular convection can be controlled by the addition of a small amount of second species, leading to a "partial-convection" state in which the convection rolls span only a part of the Hele-shaw cell, coexisting with a Leidenfrost-like state in the remaining part of the cell. The genesis of these patterns is shown to be tied to the segregation of two species along the horizontal direction which is also confirmed in particle-dynamics simulations. A possible driving mechanism for horizontal segregation under purely vertical shaking is discussed in terms of an anisotropic diffusion tensor. 


\section{EXPERIMENTAL METHOD AND SIMULATION TECHNIQUE}

The experimental setup consists of a quasi-two-dimensional rectangular Plexiglas container which is mounted on an electromagnetic shaker (Ling Dynamics System V456, Brüel \& Kjaer). The scaled length $(L / d)$, depth $(D / d)$, and height $(H / d)$ of this container are $100,5.5$, and 80 , respectively; a similar setup was used by Eshuis et al. [14] and subsequently by two of the present authors [23-25] to probe the patternformation scenario in vertically driven monodisperse granular matter. In this paper we consider a binary mixture of spherical glass and steel balls of the same diameter, $d \approx 1.0 \mathrm{~mm}$, having a density ratio of $\rho_{\text {steel }} / \rho_{\text {glass }} \approx 3.06$. The container is filled up to a specified layer depth at rest,

$$
F=\frac{h_{T}}{d}=\frac{h_{g}}{d}+\frac{h_{s}}{d} \equiv F_{g}+F_{s} \in(2.5, \ldots 10),
$$

where $h_{g}$ and $h_{s}$ are the initial depths of glass and steel balls. An equimolar (50:50) mixture of same-size particles, for which the initial layer heights of glass and steel balls are equal $\left(h_{g}=h_{s}\right)$, was used in most experiments; the effect of varying the relative number fraction of steel balls $\left[F_{s} / F=h_{s} / h_{T} \in\right.$ $(0,0.5)]$ was also assessed for a few cases.

The particle-filled container is vibrated vertically, with a harmonic wave $y=A \sin (2 \pi f t)$ of amplitude $A$ and frequency $f$ by the electromagnetic shaker which operates in a closed-loop, controlled by a controller (COMET, Brüel \& Kjaer) and an amplifier (PA 1000L, Brüel \& Kjaer) through a software interface. To generate a feedback signal of required amplitude and frequency of the harmonic excitation, a piezoelectric accelerometer (DeltaTron ${ }^{\odot}$ ) is attached on the head expander over which the Plexiglas container is mounted.

\section{A. Initial configuration and experimental protocol}

The experiments were performed for a range of shaking acceleration or intensity,

$$
\Gamma=\frac{4 \pi^{2} A f^{2}}{g} \in(0,50),
$$

in both up-sweeping (increasing frequency $f$ from the rest state) and down-sweeping (decreasing frequency from an excited state at the same ramping rate) modes. For a specified shaking amplitude $A / d(=3,6)$, the shaking acceleration $\Gamma$ was increased or decreased by increasing or decreasing frequency $(f)$ at a linear rate; all results presented correspond to a linear frequency ramping of $0.01 \mathrm{~Hz} / \mathrm{s}$; the results are found to be almost identical for a ramping rate of $0.1 \mathrm{~Hz} / \mathrm{s}$. To assess the stability of the observed patterns, many experiments were repeated at fixed $\Gamma$ for at least $30 \mathrm{~min}\left[>O\left(10^{4} \tau\right)\right.$, where $\tau=1 / f$ is the time period of shaking].

The up-sweeping experiments were done with an initial configuration of a randomly mixed mixture of steel and glass balls, and the final state of each up-sweeping experiment is used as the initial state for its down-sweeping evolution at the same rate of frequency ramping. Some experiments were also repeated with segregated initial states: (i) steel balls on top of glass balls and (ii) glass balls on top of steel balls; the reported patterns are found to be qualitatively similar irrespective of the initial state.

\section{B. Imaging and velocity measurement}

The temporal evolution of the collective motion of particles has been recorded using a high-speed camera (IDT MotionPro Y4S3) at 1000 frames/s; this camera can operate at 5100 fps at the full resolution of $1016 \times 1016$ pixels. The images were analyzed to yield information on the onset value of $\Gamma$ for different types of patterns and their characteristics (asynchronous or synchronous, segregation, and coexistence of different phases). Moreover, a commercial PIV (particle image velocimetry) software ("Dynamic Studio Software" of Dantec Dynamics A/S, Denmark) was used to extract the coarse-grained or hydrodynamic velocity field by processing the images using an adaptive-correlation technique in which the size of the interrogation window is varied adaptively from $64 \times 64$ to $16 \times 16$ pixels, with $50 \%$ overlap. The related experimental details of image analysis and the PIV methodology are documented in Ref. [23].

\section{Event-driven simulation}

Event-driven simulations for a similar system of vibrated binary mixture were carried out to cross check the robustness of the present experimental findings. A binary mixture of equal size hard inelastic spherical particles of a specified density ratio $\left(\rho_{\text {steel }} / \rho_{\text {glass }}=3.06\right)$ is used as a model granular mixture. The particles are considered as rough hard spheres with translational and rotational degrees of freedom, and the whole box is vibrated by a biparabolic sine interpolation in the same region of the phase-space of experiments; the event-driven algorithm and simulation details can be found in Ref. [26]. The collisions between particles are modelled by normal and tangential restitution coefficients: $e_{n}=e_{t}=$ 0.95 for glass-glass, $e_{n}=e_{t}=0.85$ for steel-steel, and $e_{n}=$ $e_{t}=0.9$ for steel-glass collisions; the static and dynamic friction coefficients are $\mu_{s}=\mu_{t}=0.1$ for both materials. We have checked that the qualitative trends of simulation results [presented in Figs. 1(b), 4, and 7] do not depend on our choice of different restitution coefficients for collisions of glass-glass, steel-steel, and glass-steel balls-therefore the observed patterns are not solely driven by inelasticity of particle collisions. After several initial trials, the above choice of restitution coefficients was made to qualitatively reproduce the experimental phase diagram of patterns as discussed in Sec. III A.

The collisions of particles with the bottom plate as well as with front, back, and two side walls are modelled in the same manner as for particle-particle collisions, with $e_{n}=e_{t}=0.95$ and $\mu_{t}=\mu_{d}=0.1$. We have checked that replacing the front and back walls with periodic boundary conditions does not affect the observed patterns.

\section{PATTERNS, SEGREGATION, AND CONVECTION CONTROL}

\section{A. Phase diagram of patterns: Experiment vs simulation}

We begin by describing the complete phase diagram of all patterns in the $(\Gamma, F)$ plane for up-sweeping experiments, shown in Fig. 1(a); we also refer to Supplemental Material [27] movies 1 and 2 for a visual inspection of different patterned states. The symbols in Fig. 1(a) represent the onset of a different 

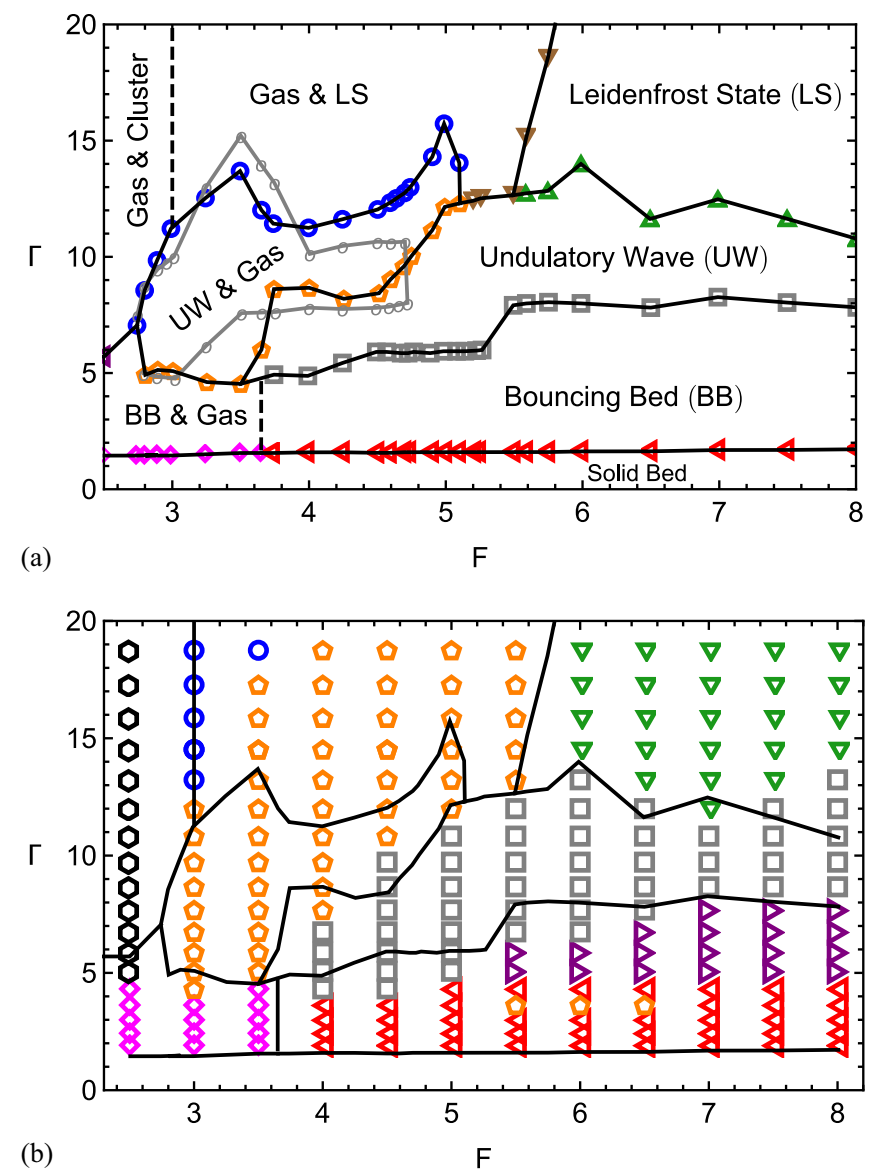

FIG. 1. (a) Phase diagram of patterns in $(\Gamma, F)$ plane for upsweeping experiments for $F_{g}=F_{s}$ (equimolar mixture) with a shaking amplitude of $A / d=3$. (See Supplemental Material [27] movies 1 and 2 for a visual inspection of different patterns.) The symbols represent the locations of transitions between two states (e.g., "BB \& Gas" and "UW \& Gas") for a specified layer depth $F$; the vertical dashed line represents an approximate phase boundary between two consecutive $F$ that represent two different states ("Gas \& Cluster" and "Gas \& LS"); the data for down-sweeping experiments (open grey symbols) for the region of "UW \& Gas" patterns are also superimposed; see text for details. (b) Phase diagram from MD simulations; the broken black lines denote the approximate phase boundaries obtained from up-sweeping experiments [same as in (a)]; refer to the text for patterns related to different symbols.

state or pattern when the shaking intensity $(\Gamma)$ is increased from zero for a specified layer depth $F$; the broken lines joining different symbols represent "approximate" phase boundaries for the transition between two patterned states in the $(\Gamma, F)$ plane. The stable nature of different types of patterns, marked in Fig. 1(a), has been verified by repeating experiments at selected values of $\Gamma$ and $F$ over a long time, $t / \tau>10^{3}$.

For $\Gamma \sim 1$, the kinetic energy of particles is not able to overcome their dead weight and hence the granular bed moves synchronously with the container without getting detached from the base plate-this is called solid bed, that occurs below the horizontal line around $\Gamma \sim 1$ in Fig. 1(a). With increasing $\Gamma>1$, the bed detaches from the base and starts bouncing like a single body, akin to a particle bouncing off a plate with zero restitution coefficient - this is the regime of bouncing bed

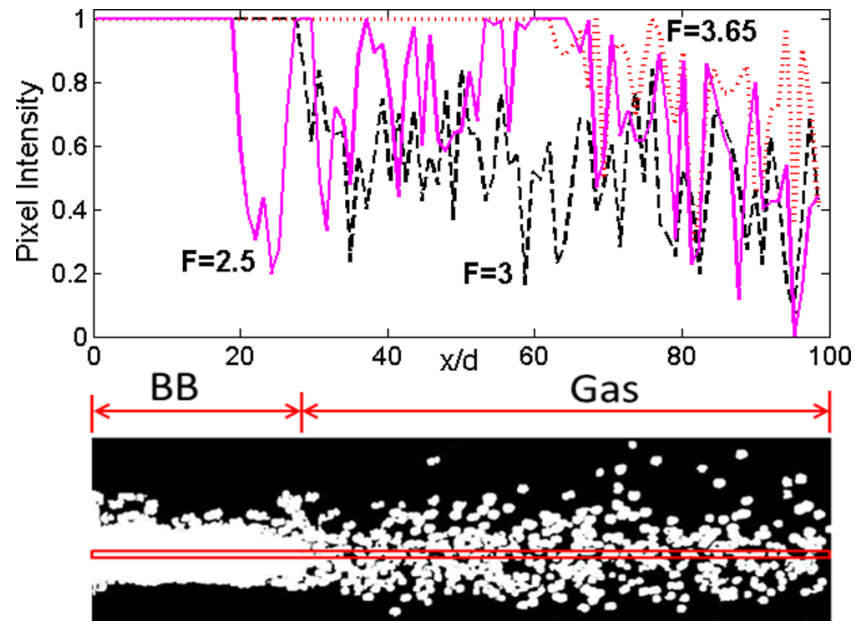

FIG. 2. Pixel-intensity profiles (upper panel) for patterns at $\Gamma=$ 3.5 for different $F$ (refer to Fig. 1); the lower panel shows the binary image of the pattern for $F=2.5$, and thin red box represents the region over which the pixel intensity is vertically averaged to yield the profiles shown in the upper panel.

(BB) whose motion is synchronized with the external shaking frequency and hence called a synchronous wave (see movie 1 in the Supplemental Material [27]). For the present system of a binary mixture, the solid bed gives birth to a BB state for $F \geqslant 3.75$, and a mixed BB-and-gas state for smaller filling heights $F<3.75$ - these two regions are marked in Fig. 1(a) above the solid-bed region.

An illustration of the BB-and-gas state is displayed in the lower panel of Fig. 2 at a shaking intensity of $\Gamma=3.5$ for a layer height of $F=2.5$. This represents a binary pixel image of the snapshot of the instantaneous particle configuration-it is seen that a relatively low-density state (populated largely by heavier steel balls) on the right of the container coexists with a bouncing-bed state on its left. The upper panel of Fig. 2 displays the average pixel-intensity profiles for $F=2.5,3$, and 3.65-these profiles have been calculated by averaging the pixel intensities over a horizontal strip (see the red-colored box in the lower panel) of height of approximately two particle diameters. It is clear that with increasing $F$ the length of the dilute gaseous region decreases, with a transition from BB and gas to a complete BB state occurring at $F>3.75$ [marked by the vertical dashed line in Fig. 1(a) for $\Gamma<5$ ].

In Fig. 1(a) the region enclosed by blue circles and orange pentagons represents another "mixed" pattern (UW and gas) consisting of a period-2 undulatory wave (UW) and a gaslike state-in this region the data for down-sweeping experiments, marked by grey circles, are also superimposed. These experiments were repeated at least three times, and we found hystereticlike behavior for the onset of the UW-and-gas pattern only at larger layer depths, i.e., $\Gamma_{\text {onset }}^{\text {up }}>\Gamma_{\text {onset }}^{\text {down }}$ for $F>4.0$. [For other patterns marked in Fig. 1(a), the onset value of $\Gamma$ at any $F$ shows little variation for up-sweeping and down-sweeping experiments.] From the Supplemental Material movie 1 [27], it can be verified that the mixed state of UW and gas maintains its shape and position for a very long time, and hence this as well as other patterns in Fig. 1(a) are stable. The characteristic features and novelty of 
the UW-and-gas pattern and its possible origin are discussed later in Sec. III B.

To check the robustness of new patterned states in Fig. 1(a), we have constructed the phase diagram of patterns based on MD simulations as shown in Fig. 1(b); the symbols represent simulation data that were obtained by running simulations at specified values of layer depth $F$ and ramping up shaking frequency (and hence increasing $\Gamma$ ) as done in experiments. It is clear that all patterns uncovered in experiments are also found in simulations, although there are variabilities in terms of the range of $(\Gamma, F)$ over which each pattern can persist in simulations. For example, the value of $\Gamma$ for the onset of the mixed-state UW and gas [denoted by orange-colored pentagons in Fig. 1(b)] agrees closely with experiments, but these patterns persist at much larger values of $\Gamma$ in simulations than in experiments. Consequently, another mixed pattern of LS and gas [the Leidenfrost-like state (LS) [16] coexisting with a gaslike state, denoted by blue circles in Fig. 1(b)] are found at much larger values of $\Gamma$ in simulations. The remaining patterns in Fig. 1(b), such as gas and cluster (black hexagons), BB and gas (pink diamonds), "BB" (denoted by left triangles), "UW" (grey squares), and "LS" (inverted green triangles) are found to encompass almost the same regions of $(\Gamma, F)$ space in simulations and experiments. [The right triangles in Fig. 1(b) at $F>5$ and $\Gamma \sim 5$ represent a subharmonic $f / 4$ wave (i.e., of period 4) found only in simulations, but not in present experiments with binary mixtures; however, such $f / 4$ waves have been reported in similar experiments with monodisperse glass or steel balls [25].] A detailed description of the LS-and-gas pattern is deferred to Sec. III C, and the onset of convection (that occurs at much higher values of $\Gamma$, not shown in Fig. 1, but see the Supplemental Material movies [27] as well as Fig. 8) and its control are discussed in Sec. III D. On the whole, the comparison between experiment and simulation in Fig. 1(b) confirms that all patterns uncovered in experiments are robust.

\section{B. Coexistence of synchronous and asynchronous states: UW and gas and gas and cluster}

Figure 3 displays four images with increasing total filling depth $\left(F=F_{g}+F_{s}\right)$ at $\Gamma \approx 9$, refer to Fig. 1 . A cluster of glass-rich balls is seen on the left of the container in Fig. 3(a) that coexists with a gaslike state of heavier steel balls on the right of the container at $F_{g}=F_{s}=1.25$-this is dubbed the "gas and cluster" pattern (which is marked in the upper-left side in the $(\Gamma, F)$ plane in Fig. 1); the cluster was also observed on the right side of the container. In either case, once formed, the cluster retains its position for a long time exceeding $O\left(10^{4} \tau\right)$ and therefore the gas and cluster is a stable pattern. At the same $F=2.5$ but with increasing $\Gamma$, the cluster size decreases, it undergoes a random oscillation, and eventually vaporizes to give birth to a granular gas at large enough $\Gamma>40$ (not shown).

When the total layer depth is increased to $F=3$, we find a coexisting pattern of (i) a granular gas on the left of the container and (ii) an undulatory wave (UW) on its right as shown in Fig. 3(b) - this is dubbed the "UW-and-gas" pattern. For even deeper beds $(F=5)$, a complete UW is found to span the whole length of the container; see Fig. 3(d). These UWs are subharmonic standing waves whose time period of

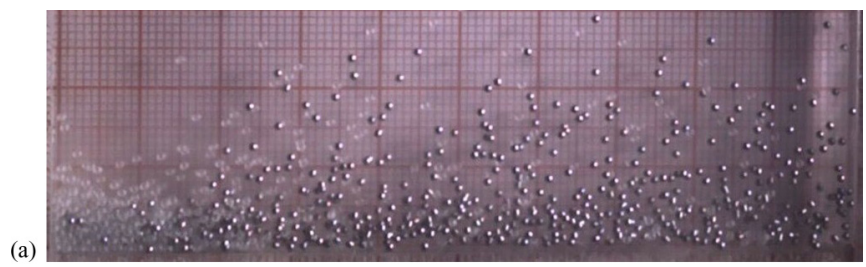

(b)

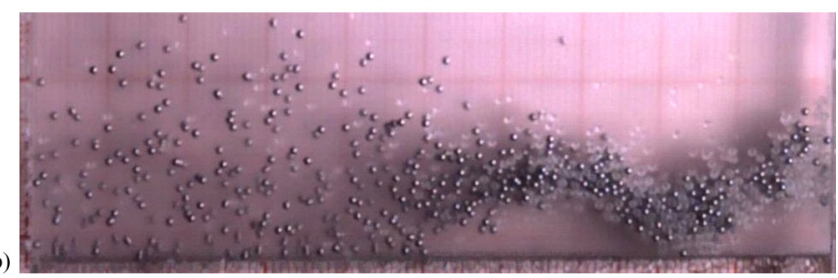

(c)

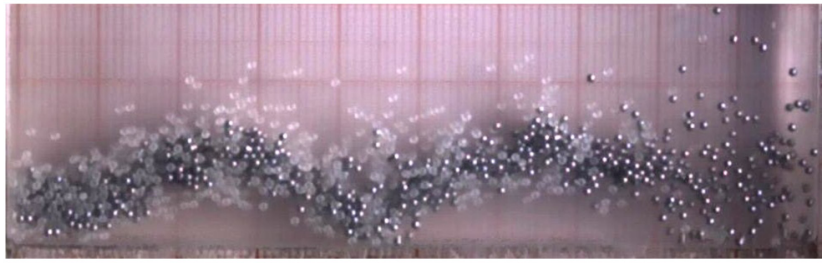

(d)

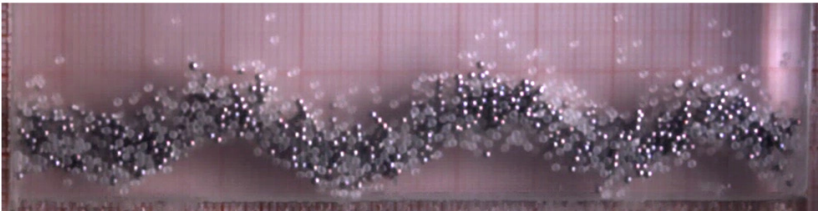

FIG. 3. Emergence of different patterns with increasing filling depth $(F)$ at $\Gamma \approx 9$. (a) gas and cluster state at $F=2.5$; (b),(c) undulatory wave (UW) and gas at (b) $F=3$ and (c) $F=4$; (d) complete UW at $F=5$. The dark and light grey particles correspond to steel and glass balls, respectively.

oscillation is twice the time period ( $\tau=1 / f$ ) of shaking (i.e., the peaks and valleys of UWs repeat after $2 \tau$; see Supplemental Material movie 1) and hence called " $f / 2$ " or "period- 2 " waves. A "partial" UW state, such as in Figs. 3(b) and 3(c), maintains its shape and position for a very long time $\left(t>5 \times 10^{4} \tau\right)-$ hence these patterns are indeed stable and long lived.

While the gas-and-cluster state [Fig. 3(a)] represents the coexistence of two phases having different spatial order (gas and liquid) and different temporal symmetry, the UW-and-gas state [Figs. 3(b) and 3(c)] represents a rare coexistence of a period-2 subharmonic wave and an asynchronous or disordered gaslike state. Such coexisting states of subharmonic or harmonic and asynchronous or disordered states having different spatial and temporal symmetries are heretofore dubbed "phasecoexisting" patterns.

In the context of vibrated "monodisperse" granular materials, the experimental work of Gotzendorfer et al. [28] reported the coexistence of subharmonic or harmonic and asynchronous (gaslike) states. However these experiments were carried out under "combined" vertical and horizontal shakings; the observed coexistence of gas and subharmonic bouncing state disappeared when the horizontal component of excitation is switched off. Another related pattern is oscillon [11] which represents a period-2 liquidlike state that coexists with a period-1 solidlike bouncing-bed state; since two parts of an oscillon are temporally synchronous (with different time periods) as well as spatially ordered, the oscillons must 
also be categorized as different from the presently uncovered phase-coexisting patterns. In contrast to the above two works, the present experiments deal with binary granular mixtures that show coexisting patterns with spontaneous horizontal segregation (Fig. 3) subject to harmonic excitation along the vertical direction. Under purely vertical vibration, we are unaware of any work where the coexistence of "synchronous" and "asynchronous" states were reported in either monodisperse or binary mixtures. The onset of coexisting synchronous and asynchronous patterns seems to be tied to the time evolution of the segregation of two species as we demonstrate below via particle-dynamics simulations of our experimental setup.

\section{Horizontal segregation in $U W$-and-gas state}

Note in Figs. 3(a)-3(c) that most of the steel balls are in a gaslike state, while the cluster and the UW are dominated by glass balls, representing a state of horizontal segregation. Since it is difficult to quantify segregation in present experiments due to the finite depth (more than five particle diameters) of our quasi-2D container, we performed event-driven simulations of the same setup to understand the role of segregation on observed patterns. The degree of segregation, along the horizontal and vertical directions, is quantified using the following order parameter [26]:

$$
\delta=1-\sum_{i} n_{g}(i) n_{s}(i) / \sqrt{\sum_{i} n_{g}^{2}(i) \sum_{i} n_{s}^{2}(i)},
$$

where $n_{g}$ and $n_{s}$ are the number density fields of glass and steel balls, respectively, in a given direction, and the sum runs from 0 to the corresponding system length in steps of one particle diameter.

The evolution of $\delta$, Eq. (3), in the UW-and-gas state is shown in Fig. 4(a) for initial (main panel) and long (left inset) times, with parameter values as in Fig. 3(b). It is observed that the vertical segregation remains small at all times and the system evolves towards a state with significant horizontal segregation at late times. The snapshots in Figs. 4(b) and 4(c) at different times confirm that the horizontal segregation precedes the formation of an UW state that coexists with a granular gas. The long-time simulation patterns are displayed in Figs. 4(c)-4(e) at time instances separated by $\tau / 2$; the wavy part indeed represents an $f / 2$ subharmonic wave that coexists with a disordered gas; notably, Figs. 4(c) and 4(e) bear a striking resemblance to experimental pattern in Fig. 3(b). Overall, the UW-and-gas patterns obtained from both experiment and simulation consist of a gaslike state coexisting with a period-2 subharmonic wave; the simulations further confirmed that the primary compositional characteristic of this mixed pattern is the horizontal segregation of two species.

The right inset in Fig. 4(a) clarifies an important point: the granular energy is unequally partitioned [29] - the heavier steel particles possess a higher kinetic energy $\left(E_{s} / E_{g} \approx 3\right)$ and hence are in a more mobile gaslike state; in contrast, the lighter glass particles possess a lower kinetic energy and are thus relatively less mobile and they tend to move together, leading to a cluster of glass-rich particles. The energy nonequipartition in a granular mixture could be a key factor for the observed seg-

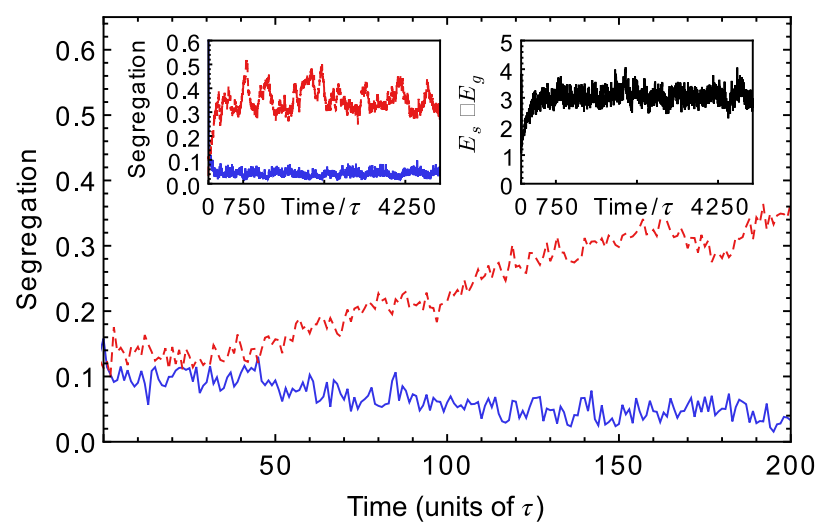

(b)
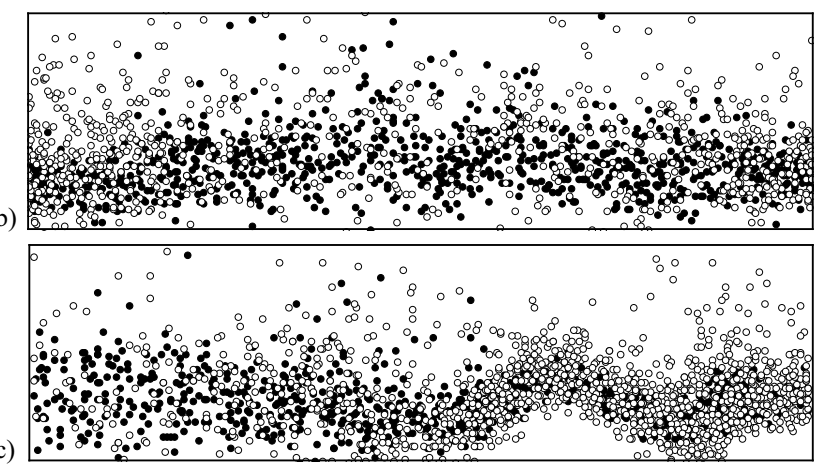

(e)

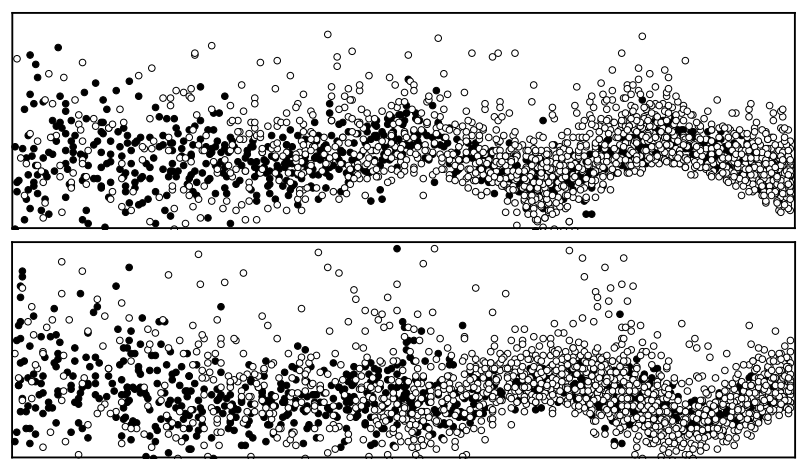

FIG. 4. Simulation data for (a) the temporal evolution of horizontal (red line) and vertical (blue line) segregation index: shortand long-time evolutions are in the main panel and the left inset, respectively. Snapshots of particles at (b) $t=100 \tau$, (c) $2500 \tau$, (d) $2500 \tau+\tau / 2$, and (e) $2501 \tau$; filled and open circles refer to steel and glass balls, respectively. Right inset in panel (a) shows the evolution of energy ratio, $E_{s} / E_{g}=\left\langle m_{s} \vec{C}_{s}^{2}\right\rangle /\left\langle m_{g} \vec{C}_{g}^{2}\right\rangle$. Parameters are as in experiments of Fig. 3(b).

regation along the horizontal direction and the resulting phasecoexisting patterns. A driving mechanism based on energy nonequipartition has been advocated for horizontal segregation observed in simulations of a submonolayer binary mixture [26] under vertical confinement, although in the latter system the resulting state appears to be akin to the gas-solid coexistence phenomenon. On the other hand, at large shaking intensities $\Gamma \sim O(10)$ of present experiments, certain Knudsen-driven rarefied effects are likely to be important, leading to anisotropic diffusion and consequently to horizontal segregation-a model for such a diffusive-mechanism is discussed in Sec. IV A. 


\section{Possible stability mechanism of partial $U W$ state}

The UW-and-gas state, such as in Figs. 3(b) and 3(c), is truly exotic since its long-time stability defies basic physics knowledge as we explain below. For monodisperse systems, the genesis of subharmonic UWs [e.g., in Fig. 3(c)] has been explained as a bifurcation from the BB state due to the bending resistance of an effective elastic bar [30]. More specifically, when a compact layer of granular materials (the BB state, constrained by two side walls) impacts on the base plate, the lowest layer of particles undergoes dilation [30] which creates an effective tension that increases with increasing $\Gamma$, thus giving birth to a buckled state of the granular layer, such as Fig. 3(c), beyond some threshold $\Gamma$. This analogy with the buckling of an elastic rod is difficult to reconcile with our finding of partial UWs [Fig. 3(b)] since the compact granular layer is free to dilate near the end where it is in constant touch with a granular gas; moreover, the "unconstrained" dry granular materials cannot sustain tensile force. For the same reason, another possible mechanism due to the subharmonic parametric instability $[9,21]$ can be ruled out for the genesis of partial undulatory waves.

A closer analysis of movies (movie 1) suggests a more complex process: when a partial UW hits the base, it dilates, resulting in expulsion of particles to the gaseous region; however, there is continual addition of "saltating" particles from the gaseous region to the UW state-both processes balance each other, leading to a stable UW-and-gas state. We speculate that the collisional pressure within the gaseous region may also play the role of a dynamic barrier, thereby helping the partial UWs to last for a long time.

\section{Leidenfrost-like states and segregation}

We now turn our attention to higher shaking intensities ( $\Gamma>20$, not shown in Fig. 1), where the system goes through different states with increasing filling depth $(F)$. At $\Gamma=50$, there is a gaseous state for $F \leqslant 3$ and this gives birth to a mixed state, Fig. 5(a) at $F=4$, of a granular gas and a cluster on the right and the left of the container, respectively. We verified that the cluster is in a Leidenfrost-like state (LS) $[14,16]$ that corresponds to a density inversion wherein a dense cloud of particles floats over a relatively dilute gaseous region of fast moving particles adjacent to the base. (This analogy of a floating granular layer with the original Leidenfrost effect [31] of a liquid drop hovering over its own vapor cushion was suggested by Eshuis et al. [16].) It has been established recently [23] that the floating dense layer has a liquidlike structure as confirmed via an analysis of its pair-correlation function, and the LS moves synchronously with the vibrating container and hence this represents a period-1 pattern. Therefore, the mixed state of gas and LS in Fig. 5(a) represents the coexistence of a disordered granular gas and a synchronous "liquidlike" period-1 wave.

A complete LS spanning the whole length of the box appears at larger filling depths (for $F>5.5$ ) as shown in Fig. 5(b). As in the case of gas-and-LS pattern, the steel and glass balls appear to be segregated along the horizontal direction in the LS pattern too-this has been verified in simulations (see below). The coarse-grained PIV velocity field (calculated by considering two successive frames separated by $1 \mathrm{~ms}$ ) within the boxed

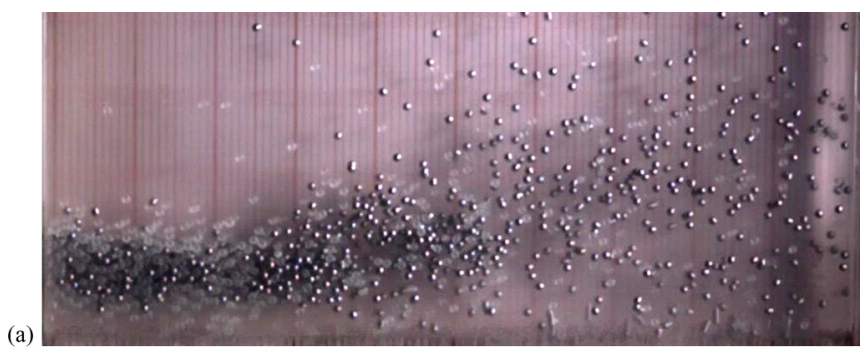

(b)

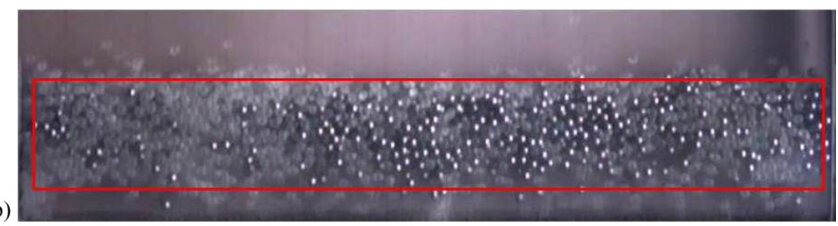

(c)

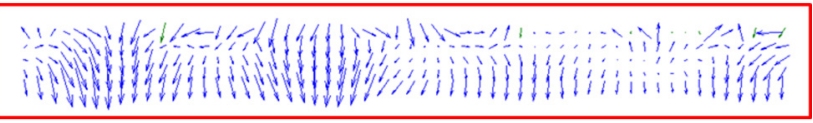

FIG. 5. Snapshots of granular Leidenfrost states (LS) and horizontal segregation: (a) LS and gas at $\Gamma=50.22(f=64.5 \mathrm{~Hz})$ and $F=4$ and (b) complete LS at $\Gamma=50.7(f=65 \mathrm{~Hz})$ and $F=6$. (c) Coarse-grained PIV velocity field for the boxed region in (b).

region of Fig. 5(b) is shown in Fig. 5(c) - there are strong correlated motions along both horizontal and vertical directions. Such correlated motion constitutes one characteristic feature of the Leidenfrost state that distinguishes it from the BB state [23] as it is evident from a comparison of Fig. 5(c) with the velocity-vector plots in Fig. 6. Figure 6 shows three snapshots of the bouncing-bed state at times $t=0, \tau / 2$, and $\tau$-each raw image of particle configuration is accompanied by its instantaneous PIV-velocity field that has been evaluated within the red box. It is seen that there is negligible lateral correlation of velocity and the particle motion is primarily correlated with the vertical motion of the box as expected for a BB state.

The simulation analog of Figs. 5(a) and 5(b) are displayed in Figs. 7(a) and 7(b), respectively. As in experiments [Fig. 5(a)], the simulations confirmed that the partial Leidenfrost state [Fig. 7(a)] is a mixed pattern of a gaslike state dominated by heavier steel balls that coexists with a glass-rich Leidenfrostlike state. A closer inspection of Fig. 7(b) indicates that there is segregation of steel and glass balls along the horizontal direction for the case of the "complete" Leidenfrost state too. For the latter case, the temporal evolution of segregation index, Eq. (3), obtained from simulations is displayed in Fig. 7(c). There is clear vertical segregation for a short time $(t / \tau<200)$, but the system eventually reaches a steady state with significant horizontal segregation. We therefore conclude that both the partial and complete Leidenfrost states are characterized by the segregation of steel and glass balls along the horizontal direction.

\section{Granular convection and its control}

For the same shaking intensity of $\Gamma \approx 50$ as in the Leidenfrost state in Fig. 5(b), the corresponding monodisperse system of glass balls showed a convective motion [14], containing six counterrotating rolls which can be visualized from the Supplemental Material [27] movie 2. This indicates a strong 

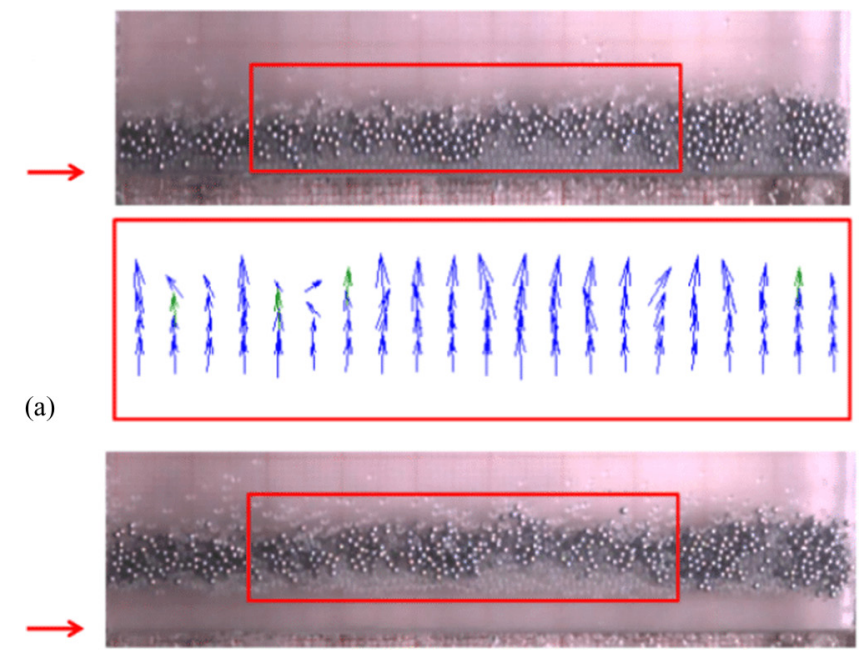

(b)
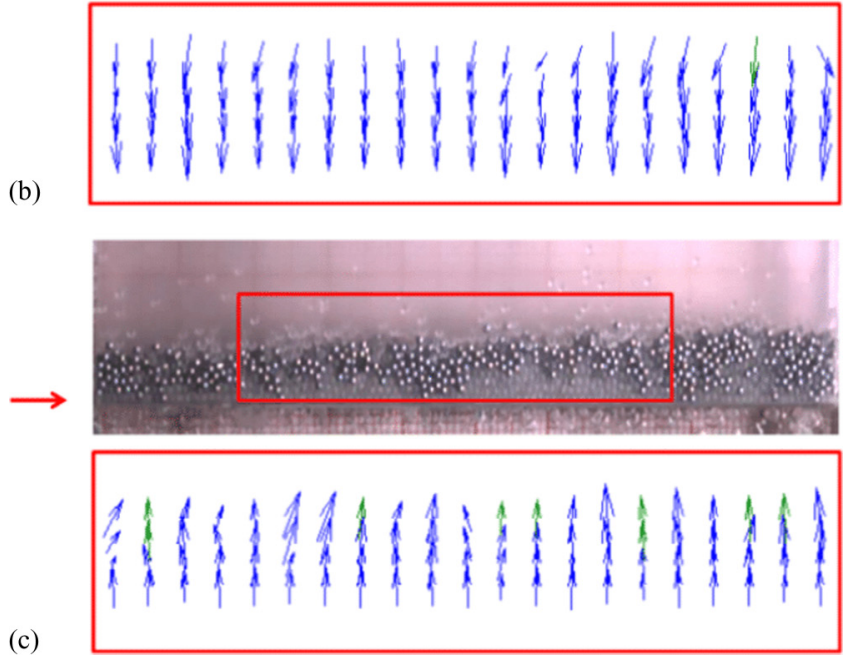

FIG. 6. Snapshot (upper panel) and PIV-velocity field (lower panel) of the bouncing-bed (BB) states at $\Gamma=4$ and $F=4$ : (a) $t=0$, (b) $\tau / 2$, and (c) $\tau$, with $\tau=1 / f$ being the time period of harmonic shaking. The horizontal arrow in each snapshot indicates the location of the bottom of the box.

influence of steel particles on the dynamics of the mixture. To better understand this, we progressively varied the concentration of steel particles. With the addition of $1 \%$ steel balls $\left(F_{s} / F=0.01\right)$, the convection pattern and the number of rolls remain the same; this is evident in the PIV velocity field shown in the lower panel of Fig. 8(a), with its upper panel representing the corresponding raw image of particle configuration. The velocity has been calculated within the red-boxed region of the raw image - this represents an instantaneous velocity field, calculated over two frames separated by $1 \mathrm{~ms}$; however, due to the small number of particles in the system, the accuracy of the calculated velocity field is limited. Here we are interested only in the gross features of the hydrodynamic velocity field, i.e., whether it contains a circulating motion or not. It may be noted that the convection rolls, such as those in Fig. 8(a), represent the granular analog of the well-studied "buoyancy-induced" thermal convection which was unequivocally demonstrated in experiments of Eshuis et al. [14].

Figures 8 (b)-8(d) show a surprising effect-the number of rolls decreases to 4 at $F_{s} / F=0.02$ [Fig. 8(b)] and to two at (a)

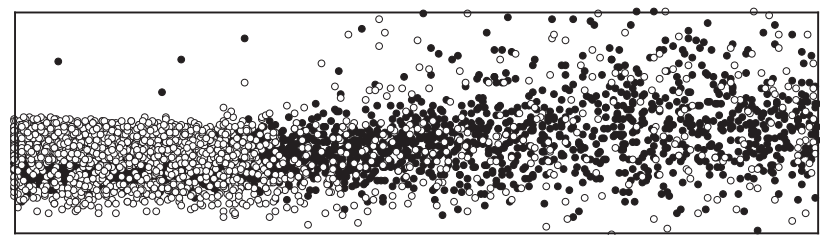

(b)
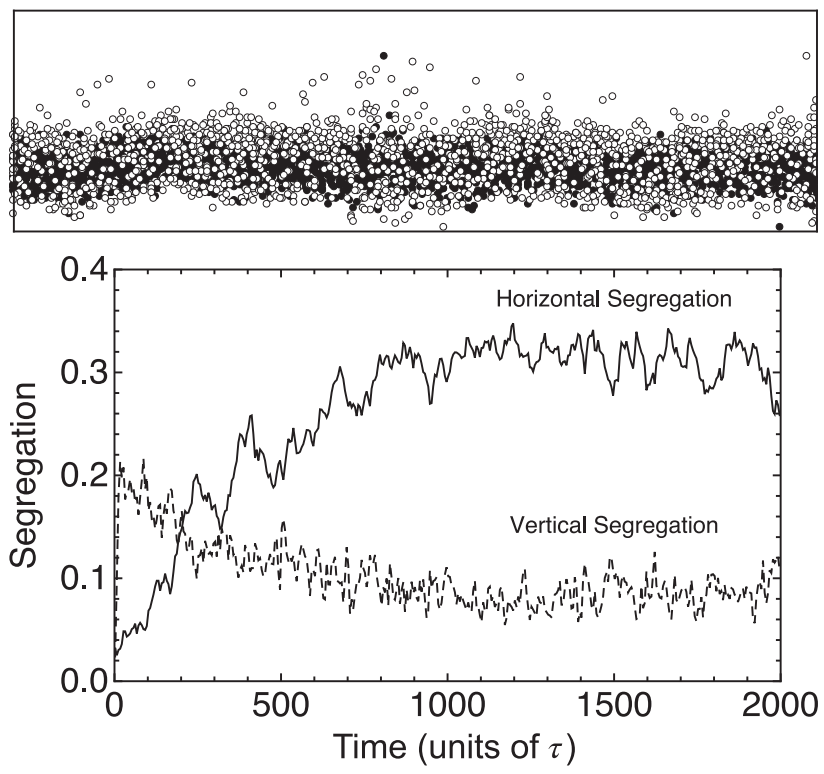

FIG. 7. Simulation snapshots of granular Leidenfrost states (LSs) and horizontal segregation: (a) LS and gas at $F=4$ and (b) complete LS at $F=6$; other parameter values are as in Figs. 5(a) and 5(b), respectively; filled and open circles refer to steel and glass balls, respectively. (c) Simulation data for the evolution of horizontal (upper solid line) and vertical (lower dashed line) segregation indices for parameter values of (b).

$F_{s} / F \geqslant 0.05$ [Figs. 8(c) and 8(d)]. In the latter two cases, a "complete" convection state, spanning the whole length of the container, ceases to exist and the system degenerates into a partial convection state, characterized by a pair of counterrotating rolls in one side of the container and a Leidenfrost state on the other side. An inspection of raw images in Fig. 8 indicates that the convective rolls in binary mixtures are populated by steel balls, and the remaining part is dominated by lighter glass balls which represents a liquidlike [see the velocity field on the right side of each image in panels (c) and (d)] Leidenfrost state. The inspection of Supplemental Material movie 2 reveals that the convective motion within a partialconvection state is spatially nonuniform and unsteady, coupled with occasional "jetlike" expulsion of particles. The present discovery of partial-convection states, such as in Figs. 8(c) and 8(d), firmly establishes that the "side walls" [12] are not required for the onset of "buoyancy-driven" $[13,14,23,32,33]$ granular convection.

The effect of the relative number fraction $\left(F_{s} / F\right)$ on convection is summarized in Fig. 9. For a specified shaking intensity (say, $\Gamma=50$ in Fig. 9), complete convection is possible only for a very small range of $F_{s} / F \leqslant 0.04$, the partial-convection states occur for $F_{S} / F \in(0.05,0.45)$ which eventually degenerate into a complete Leidenfrost state for $F_{s} / F>0.45$ [see Fig. 5(b) for an equimolar mixture]. The 


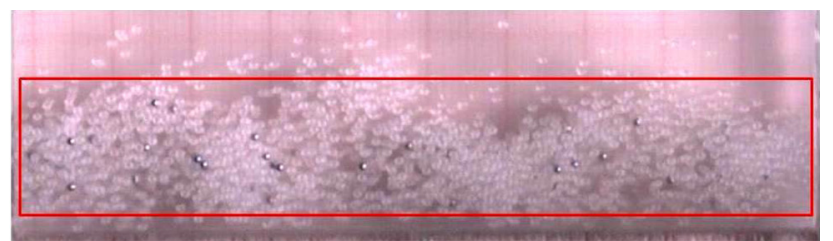

(a)
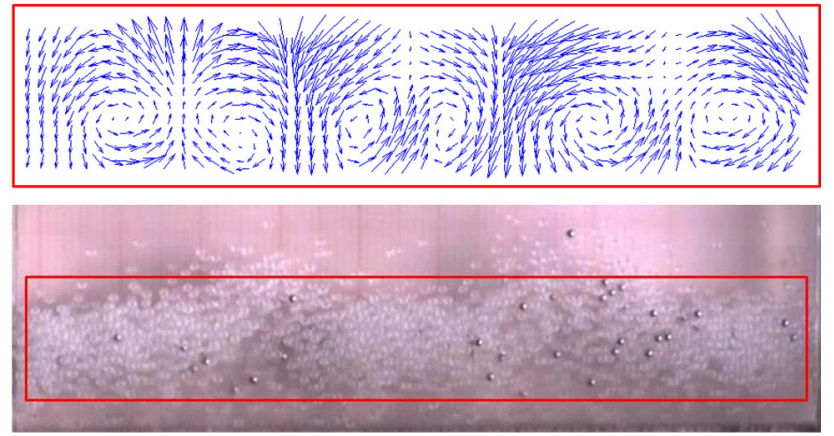

(b)

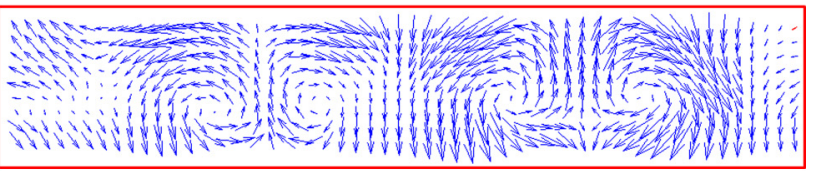

(c)
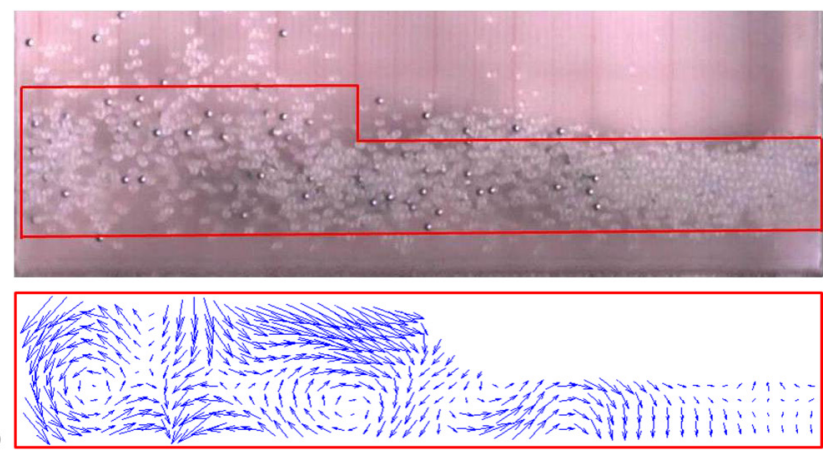

(d)

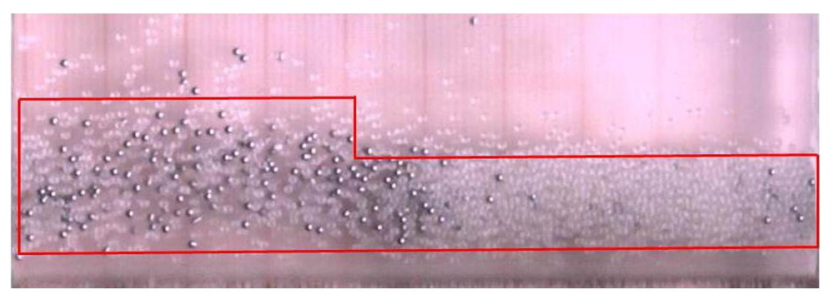

FIG. 8. Instantaneous snapshot (upper panel) and its coarsegrained PIV velocity field (lower panel) for mixtures of glass and steel balls with (a) $1 \%$, (b) $2 \%$, (c) $5 \%$, and (d) $10 \%$ steel balls for $\Gamma \approx 50, A / d=3$, and $F=F_{g}+F_{s}=6$. The velocity field (lower panel) is calculated within the boxed region (upper panel) of each snapshot.

red-diamond symbols on the left of Fig. 9 indicate that the onset value of $\Gamma$ for convection decreases sharply with increasing steel balls $\left(F_{S} / F\right)$ up to $F_{S} / F \leqslant 0.04$ and increases thereafter: therefore, the heavier particles play a dual role of enhancing and suppressing the convection intensity. The delayed convection beyond a critical value of $F_{S} / F(\geqslant 0.04)$ is presumably

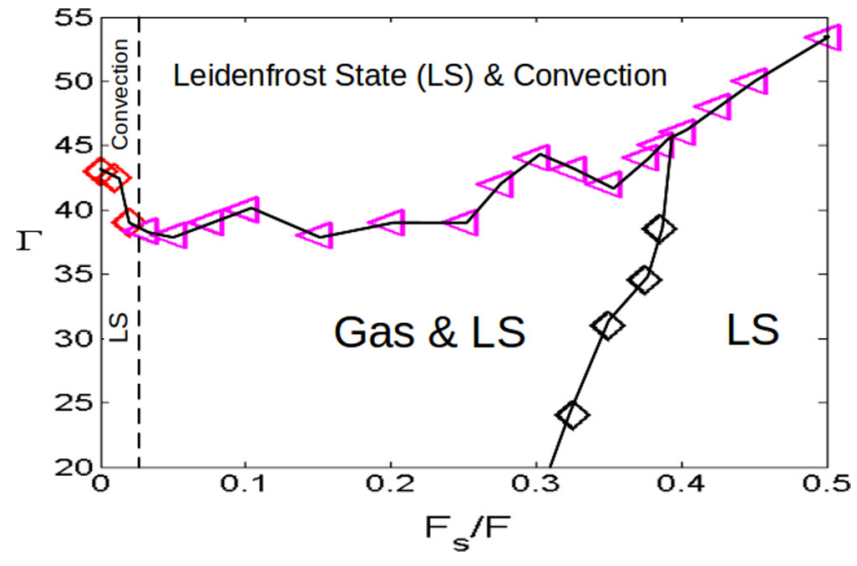

FIG. 9. Effect of relative number fraction of steel balls on the phase diagram of patterns in $\left(\Gamma, F_{s} / F\right)$ plane for $F=F_{g}+F_{s}=6$ and $A / d=3$; other details are as in Fig. 1. The vertical dashed line (at $F_{S} / F \approx 0.04$ ) represent an approximate phase boundary between two states ["convection" and "LS and convection"; "LS (Leidenfrost state)" and "gas and LS"].

due to the nonuniform fluidization of two species. The issue of nonuniform fluidization can be tied to the unequal partition of granular energy between steel and glass balls $\left(E_{s} \neq E_{g}\right)$ as confirmed in present simulations too. In summary, (i) the multitude of pattern transitions (among various mixed states) appearing from the rest state of a solid bed with increasing $\Gamma$ and varying total fill height $F$, (ii) the partial convection state, and (iii) the recipe for convection control by varying the relative number fraction of two species are reported here.

The possibility of convection suppression and its underlying mechanism have been reported in a simulation study [33] with binary granular mixtures to which we compare our results below. In particular these authors [33] showed that adding about $12 \%$ of quasielastic particles (of species 2 with its restitution coefficient $e_{22}=0.9992$ ) in an otherwise inelastic system (of species 1 with $e_{11}=0.96$ ) can completely suppress the convection rolls to a gaslike state for a specified shaking intensity (see their Figs. 11 and 12), pointing towards the crucial role of inelastic dissipation. Note that their simulations correspond to extremely high shaking intensity $\Gamma=1250$ with an initial fill height of $F \approx 19.5$ (and the mean volume fraction of particles is about 0.0008 ) and they did not report either partial convection or Leidenfrost-like states or horizontal segregation. In our experiments, the restitution coefficients of steel and glass balls are nearly equal $\left(e_{s s} \approx e_{g g} \approx 0.9\right)$, and hence the present transition scenario is unlikely to be driven solely by the difference in inelasticities of two species; rather the combined effects of inelasticity and mass disparity in an otherwise "nondilute" binary mixture $[29,34,35]$ is expected to lead to nonuniform fluidization of two species which might be responsible for the observed transition route of "convection $\rightarrow$ partial convection $\rightarrow$ LS" at fixed $\Gamma(>40)$ with increasing $F_{s} / F$ (see Fig. 9).

Juxtaposing the "convection $\rightarrow$ gas" transition of Ref. [33] with Fig. 9 at large enough $\Gamma$, we note that the work of Ref. [33] belongs to a "dilute" granular gas $(\Gamma=1250)$ for which we have no experimental data $(\Gamma<55)$ to compare with. Nevertheless, the simulation transition can be explained by 
considering an effective restitution coefficient [i.e., $e_{\text {eff }}=$ $\left(e_{11}+e_{22}\right) / 2$ for an equimolar mixture] for the binary mixture such that the control parameter [i.e., the inelasticity parameter $q N=N\left(1-e_{\text {eff }}\right) / 2$ [32] which is equivalent to the Rayleigh number and hence acts as a driving force for the onset of convection from a gaslike state] decreases with the addition of quasielastic particles. Therefore, the convection-suppression mechanism of Ref. [33] is tied with the inelasticity-driven transition in a granular gas (under some temperature gradient) where a gaslike state gives rise to convection rolls with increasing inelasticity $[32,36]$ and vice versa-this constitutes a fundamental difference of Ref. [33] with our finding of convection $\rightarrow$ partial convection $\rightarrow$ LS with increasing the number density of a second species at fixed shaking intensity $\Gamma$.

\section{DISCUSSION AND CONCLUSION}

\section{A. Spontaneous horizontal segregation and anisotropic diffusion}

The present experiments and simulations confirmed that most patterns at large shaking intensities (Figs. 2-5, 7, and 8 ) are characterized by the segregation of steel and glass balls along the horizontal direction, with the heavier steel balls being in a gaslike disordered or a convective state and the lighter glass balls in a liquidlike UW, cluster, or Leidenfrost state. At this point we must emphasize that our finding of "spontaneous" horizontal segregation [e.g., Figs. 3(a)-3(c)] under vertical vibration is distinctly different from those of Ref. [37]. In the latter and related works, the base of the container had an asymmetric "sawtooth" shape which acts like a ratchet for horizontal transport, resulting in "preferred" segregation along the horizontal direction. In contrast, the present finding of horizontal segregation is not boundary driven; rather it appears spontaneously under vertical excitations when the shaking intensity is large enough $[\Gamma \sim O(10)$ or more $]$ and the fill height is small enough $[F \sim O(5)$ or less].

If the segregation of two species along the horizontal direction is responsible for observed coexisting patterns (such as in Fig. 3), the length $(L / d)$ of the container must be sufficiently large to allow horizontal segregation to take place, otherwise the two species are expected to be well mixed along the nondriven (horizontal) direction. This is indeed the case as demonstrated in Fig. 10. While the larger container $(L / d=50)$ in Fig. 10(a) represents a horizontally segregated state, the smaller container $(L / d=20)$ in Fig. $10(\mathrm{~b})$ is a gaslike state with no visible horizontal segregation; for the same parameter values of Fig. 10, increasing the container length to $L / d=100$ leads to another coexisting pattern of UW-and-gas state (see Fig. 1). It is therefore clear that the length of the Hele-Shaw cell must be large enough for the appearance of phase-coexisting patterns (with two species being segregated along the horizontal direction) as found in Fig. 1.

Here we argue that certain Knudsen-driven rarefied effects become important with increasing shaking intensity $\Gamma$, leading to anisotropic diffusion which can drive horizontal segregation under purely vertical shaking as we illustrate below; our proposed mechanism for horizontal segregation is based on well-known facts about rarefied gas kinetic theory [38-41]. In a driven binary mixture, the net diffusion velocity between two (a)

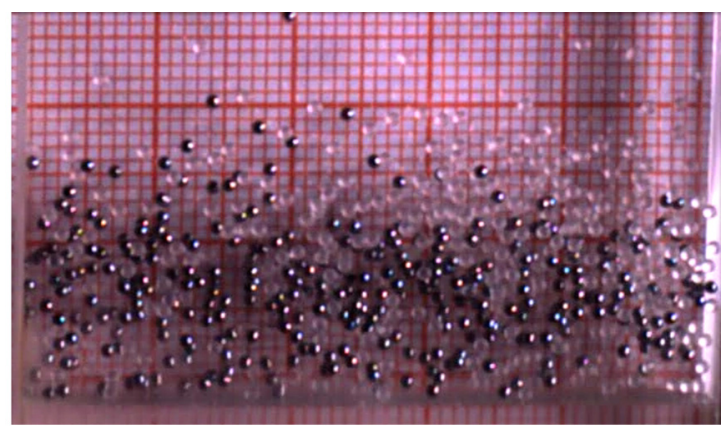

(b)

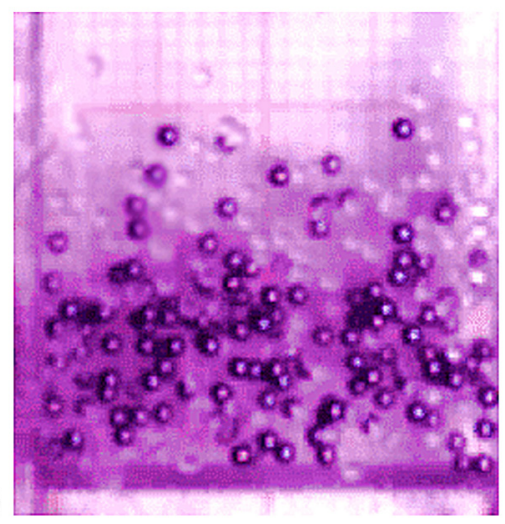

FIG. 10. Effect of container length on patterns: (a) $L / d=50$ (a horizontally segregated state with a "glass-rich" cluster is seen on the right of the container) and (b) $L / d=20$ (a gaslike nearly homogeneous state) for $\Gamma=7, F_{g}=F_{s}=1.5$, and $A / d=3$.

species $A$ and $B$ is given by $[41,42]$

$$
\boldsymbol{v}^{A B}=\boldsymbol{v}^{A}-\boldsymbol{v}^{B}=-\frac{n}{n^{A} n^{B}} \boldsymbol{D}^{A B} \cdot[\alpha \nabla n+\beta \nabla T],
$$

where $n=n^{A}+n^{B}$ is the total number density of the mixture, and the expressions for $\alpha$ and $\beta$ can be obtained from kinetic theory; the most general form of the diffusion tensor (in twodimensions) is

$$
\boldsymbol{D}^{A B}=\left(\begin{array}{ll}
\mathcal{D}_{x x} & \mathcal{D}_{x y} \\
\mathcal{D}_{y x} & \mathcal{D}_{y y}
\end{array}\right) .
$$

At Navier-Stokes order (when the gradients of hydrodynamic fields are small, which is characterized by "small" values of the Knudsen number, $\mathrm{Kn} \sim 0$ ), it is well known that $\mathcal{D}_{x y}=0=$ $\mathcal{D}_{y x}$ and $\mathcal{D}_{x x}=\mathcal{D}_{y y}=\mathcal{D}_{0}$ and the scalar diffusion coefficient of a binary gas is given by [41,42]

$$
\mathcal{D}_{0}=\frac{1}{2 n d^{A B}}\left(\frac{m^{A B} T}{2 \pi m^{A} m^{B}}\right)^{1 / 2} \propto \sqrt{T},
$$

where $d^{A B}=d^{A}+d^{B}, m^{A B}=m^{A}+m^{B}$, and the $T$ is the mixture temperature.

When the Knudsen number deviates significantly from zero, the higher-order gradients become important which can be described by Burnett-order theories [38,39]. Drawing an analogy between mass transport and heat transport, the form of the anisotropic diffusion tensor, Eq. (5), can be justified [43], with $\left(\mathcal{D}_{x y}, \mathcal{D}_{y x}\right) \neq 0$ and $\mathcal{D}_{x x} \neq \mathcal{D}_{y y}$; it has been established [43] that the off-diagonal terms of the conductivity or diffusion tensor appear at the second order in a gradient expansion [i.e., at $O\left(\mathrm{Kn}^{2}\right)$ ]. Assuming that the anisotropic diffusion tensor is 
given by Eq. (5) and under purely vertical shaking along the $y$ direction as in our experiments, the horizontal component of the diffusion velocity follows from Eq. (4):

$$
v_{x}^{A B}=\alpha \mathcal{D}_{x y} \frac{\partial n}{\partial y}+\beta \mathcal{D}_{x y} \frac{\partial T}{\partial y} .
$$

It is clear that the $v_{x}^{A B}$ is nonzero if and only if the off-diagonal components of the diffusion tensor are nonzero and therefore the vertical gradients alone (in density and/or temperature) can drive segregation of two species along the horizontal direction. This provides a tentative explanation for the observed "spontaneous" horizontal segregation in our setup. A detailed theory (including the dependence of the "critical or minimum" container length for the sustenance of horizontal segregation on the initial layer height $F=h_{T} / d$, the shaking intensity $\Gamma$, and the diffusion coefficients) can be worked out once all components of the Burnett-order diffusion tensor and other related transport coefficients are known, which is left to a future work.

\section{B. Conclusions and outlook}

Using both experiments and simulations, we uncovered Chimera-like patterns in vertically vibrated binary granular mixtures that are characterized by the coexistence of two phases (see Supplemental Material movie 1 [27]): (i) a disordered or asynchronous granular gas coexisting with (i a) a subharmonic (period 2) undulatory wave, (i b) a synchronous (period 1) cluster, and (i c) a Leidenfrost-like state (of period 1 [23]); and (ii) a Leidenfrost state coexisting with a pair of rolls (i.e., a partial convection state). The coexistence of time-periodic synchronous liquidlike and disordered or asynchronous gaslike states is dubbed "phase-coexisting" patterns - these mixed patterns are made of two parts having different temporal and spatial symmetries. Both experiments and simulations confirmed that the onset of all mixed patterns is a consequence of the horizontal segregation of heavier and lighter particles. We discovered a giant effect of adding a small amount of heavier particles that can control (i) the onset of granular convection, (ii) the number of convection rolls, and (iii) the partial convection state (see Supplemental Material movie 2 [27]). On the whole, controlling patterns in granular flows using such a simple recipe may have far reaching consequences from the viewpoint of potential applications in processing industries.

The segregation between heavier and lighter particles along the horizontal direction is shown to be the key factor for all observed patterns as well as for the protocol for convection control. A possible mechanism for "spontaneous" horizontal segregation under vertical vibration has been put forward in terms of an "anisotropic" diffusion tensor-such a diffusive mechanism is likely to be operative at high-shaking intensities such that the heavier species stays in a relatively rarefied or dilute state compared to the lighter species. Increasing the lateral confinement (i.e., by decreasing the length $L$ of the container) leads to patterns in which two species appear well mixed, which suggests that the diffusion of two species along the horizontal direction indeed plays a key role on the genesis of horizontal segregation, resulting in a variety of phasecoexisting "Chimera-like" patterns that we have uncovered. The derivation of the exact form of the diffusion tensor and the underlying analyses are beyond the scope of the present work and left to the future.

\section{ACKNOWLEDGMENTS}

We are grateful for the funding support from the Department of Atomic Energy, Government of India (via DAE-SRC Outstanding Research Investigator Award to M.A., Ref. No. 2010/21/06-BRNS/2122). We sincerely thank S. Bharadwaj for help with PIV analysis of some data, and Prof. S. Luding for comments on the manuscript.

Experiments were carried out by I.H.A. and the numerical simulations by N.R.
[1] L. R. Brown, Fundamental principles of segregation, J. Inst. Fuel 13, 15 (1939).

[2] A. Kudrolli, Size separation in vibrated granular matter, Rep. Prog. Phys. 67, 209 (2000).

[3] J. M. Ottino and D. V. Khakhar, Mixing and segregation of granular materials, Annu. Rev. Fluid Mech. 32, 55 (2000).

[4] A. Rosato, K. J. Strandburg, F. Prinz, and R. H. Swendsen, Monte Carlo Simulation of Particulate Matter Segregation, Phys. Rev. Lett. 58, 1038 (1987).

[5] T. Shinbrot and F. J. Muzzio, Reverse Buoyancy in Shaken Granular Beds, Phys. Rev. Lett. 81, 4365 (1998).

[6] A. P. Breu, H. M. Ensner, C. A. Kruelle, and I. Rehberg, Reversing the Brazil nut effect: Competition between Percolation and Condensation, Phys. Rev. Lett. 90, 014302 (2003).

[7] D. C. Hong, P. V. Quinn, and S. Luding, Reverse Brazil nut Problem: Competition between Percolation and Condensation, Phys. Rev. Lett. 86, 3423 (2001).
[8] E. Clément, J. Duran, and J. Rajchenbach, Experimental Study of Heaping in a Two-Dimensional Sand Pile, Phys. Rev. Lett. 69, 1189 (1992).

[9] S. Douady, S. Fauve, and C. Laroche, Subharmonic instabilities and defects in a granular layer under vertical vibrations, Europhys. Lett. 8, 621 (1989).

[10] M. Faraday, On a peculiar class of acoustical figures; and on certain forms assumed by groups of particles upon vibrating elastic surfaces, Philos. Trans. R. Soc. London 121, 299 (1831).

[11] P. B. Umbanhower, F. Melo, and H. L. Swinney, Localized excitations in a vertically vibrated granular layer, Nature (London) 382, 793 (1996).

[12] J. A. C. Gallas, H. J. Herrmann, and S. Sokolowski, Convection Cells in Vibrating Granular Media, Phys. Rev. Lett. 69, 1371 (1992).

[13] R. D. Wildman, J. M. Huntley, and D. J. Parker, Convection in Highly Fluidized Three-Dimensional Granular Beds, Phys. Rev. Lett. 86, 3304 (2001). 
[14] P. Eshuis, K. van der Weele, D. van der Meer, R. Bos, and D. Lohse, Phase diagram of vertically shaken granular matter, Phys. Fluids 19, 123301 (2007).

[15] P. Eshuis, D. van der Meer, M. Alam, H. J. van Gerner, K. van der Weele, and D. Lohse, Onset of Convection in Strongly Shaken Granular Matter, Phys. Rev. Lett. 104, 038001 (2010).

[16] P. Eshuis, K. van der Weele, D. van der Meer, and D. Lohse, Granular Leidenfrost Effect: Experiment and Theory of Floating Particle Clusters, Phys. Rev. Lett. 95, 258001 (2005).

[17] B. Meerson, T. Pöschel, and Y. Bromberg, Close-Packed Floating Clusters: Granular Hydrodynamics Beyond a Freezing Point?, Phys. Rev. Lett. 91, 024301 (2003).

[18] T. Pöschel and H. J. Herrmann, Size segregation and convection, Europhys. Lett. 29, 123 (1995).

[19] N. Burtally, P. J. King, and M. R. Swift, Spontaneous air-driven separation in vertically vibrated fine granular mixtures, Science 295, 1877 (2002).

[20] J. S. Olafsen and J. S. Urbach, Clustering, Order, and Collapse in a Driven Granular Monolayer, Phys. Rev. Lett. 81, 4369 (1998).

[21] I. S. Aranson and L. S. Tsimring, Patterns and collective behavior in granular media: Theoretical concepts, Rev. Mod. Phys 78, 641 (2006).

[22] P. Shukla, I. H. Ansari, D. van der Meer, D. Lohse, and M. Alam, Nonlinear instability and convection in vertically vibrated granular bed, J. Fluid Mech. 761, 123 (2014).

[23] I. H. Ansari and M. Alam, Pattern transition, microstructure and dynamics in vertically vibrated granular bed, Phys. Rev. E 93, 052901 (2016).

[24] I. H. Ansari and M. Alam, Exotic patterns and convection control in a vibrated bed of binary granular mixtures, Bull Am. Phys. Soc. 58, 300 (2013).

[25] I. H. Ansari and M. Alam, Patterns and velocity field in vertically vibrated granular materials, in Powders and Grains 2013: Proceedings of the 7th International Conference on Micromechanics of Granular Media, edited by A. Yu, K. Dong, R. Yang, and S. Luding, AIP Conf. Proc. No. 1542 (AIP, Melville, NY, 2013), pp. 775-778.

[26] N. Rivas, P. Cordero, D. Risso, and R. Soto, Segregation in quasitwo-dimensional granular systems, New J. Phys. 13, 055018 (2011).

[27] See Supplemental Material at http://link.aps.org/supplemental/ 10.1103/PhysRevE.97.012911 for two movies. While movie 1 displays temporal evolution of a phase-coexisting state of "UW and gas" at a shaking intensity of $\Gamma=9$ and a layer height $F=3$ for an equimolar binary mixture, movie 2 displays the transition scenario "convection to partial-convection to Leidenfrost state" with increasing relative number density $\left(F_{s} / F\right)$ of steel balls for nonequimolar mixtures at given values of $\Gamma=50$ and $F=$ $F_{s}+F_{g}=6$.

[28] A. Götzendorfer, C. A. Kruelle, I. Rheberg, and D. Svensek, Localized Subharmonic Waves in a Circularly Vibrated Granular Bed, Phys. Rev. Lett. 97, 198001 (2006).

[29] K. Feitosa and N. Menon, Breakdown of Energy Equipartition in a 2D Binary Vibrated Granular Gas, Phys. Rev. Lett. 88, 198301 (2002).

[30] O. Sano, Dilatancy, buckling, and undulations on a vertically vibrating granular layer, Phys. Rev. E 72, 051302 (2005).

[31] J. G. Leidenfrost, De Aquae Communis Nonnullis Qualitatibus Tractatus (University of Duisburg, Duisburg, Germany, 1756).

[32] R. Ramirez, D. Risso, and P. Cordero, Thermal Convection in Fluidized Granular Systems, Phys. Rev. Lett. 85, 1230 (2000).

[33] D. Paolotti, A. Barrat, U. M. B. Marconi, and A. Puglisi, Thermal convection in monodisperse and bidisperse granular gases: A simulation study, Phys. Rev. E 69, 061304 (2004).

[34] M. Alam and S. Luding, How good is the equipartition assumption for the transport properties of a granular mixture?, Granular Matter 4, 139 (2002).

[35] M. Alam and S. Luding, Energy nonequipartition, rheology and microstructure in sheared bidisperse granular mixtures, Phys. Fluids 17, 063303 (2005).

[36] E. Khain and B. Meerson, Onset of thermal convection in a horizontal layer of granular gas, Phys. Rev. E 67, 021306 (2003).

[37] X. Shi, G. Miao, and H. Zhang, Horizontal segregation in a vertically vibrated binary granular system, Phys. Rev. E 80, 061306 (2009).

[38] D. Burnett, The distribution velocities in a slightly non-uniform gas, Proc. London Math. Soc. 39, 385 (1935).

[39] H. Grad, On the kinetic theory of rarefied gases, Commun. Pure Appl. Math. 2, 331 (1949).

[40] M. N. Kogan, Rarefied Gas Dynamics (Plenum, New York, 1969).

[41] S. Chapman and T. G. Cowling, The Mathematical Theory for Non-Uniform Gases (Cambridge University Press, Cambridge, England, 1970).

[42] E. Goldman and L. Sirovich, Equations for gas mixtures, Phys. Fluids 10, 1928 (1967).

[43] S. Saha and M. Alam, Non-Newtonian stress, collisional dissipation and heat flux in the shear flow of inelastic disks: A reduction via Grad's moment method, J. Fluid Mech. 757, 251 (2014). 